\title{
Competition in Mechanisms
}

\author{
MALLESH M PAI \\ Northwestern University
}

\begin{abstract}
We consider settings where sellers compete by announcing auctions or mechanisms. We sketch why, in our opinion, it is important to understand and survey some of the main results to date. We then describe recent results in 2 settings. We conclude with a discussion of directions for future progress.

Categories and Subject Descriptors: J.4 [Computer Applications]: Social and Behavioral Sciences-Economics

General Terms: Economics, Theory, Mechanisms

Additional Key Words and Phrases: Competition, Efficiency
\end{abstract}

\section{INTRODUCTION}

An old story recounts the attempts of a mathematician to ascertain the resting position of a chair with $n$ legs. While he figures out the cases of $n=1$ and $n=\infty$, the case of $n=2$ proves to be much more troublesome. Auction theory has followed the same path as our apocryphal mathematician. The case of a monopolist seller and perfect competition among sellers are both well understood. Much less is known for the case of two competing sellers, or more generally, small and finite numbers of sellers.

Oligopolistic competition is often better modeled thusly than by standard price or quantity competition. We provide two examples: (1) the major search engines sell ad slots to advertisers by each choosing a mechanism and receiving bids, and (2) competition among airlines for passengers comes down to each airline choosing a revenue management system.

The reason for the scarcity of results on oligopolistic sellers is easy to explain, if hard to resolve. There are two major impediments.

(1) Infinite Regress: When considering, for example, price competition among sellers, it is easy to see what the space of strategies for sellers should be, i.e. just the set of possible prices each can offer. By contrast, in competition in mechanisms, it is perfectly reasonable to consider a mechanism which depends on the mechanism announced by the other seller. Of course, this leads to an infinite regress. Even if a fixed point exists, it is hard to identify, and equally hard to work with.

(2) No Analog to the Revelation Principle: To compute the revenue of a given auction requires a seller to first compute the equilibrium strategies of buyers. Maximizing revenue ostensibly requires an optimization program that has a fixed point computation subroutine. The revelation principle allows us to abstract away from strategic behavior among buyers and reduces the problem of

Author's address: mallesh.pai@gmail.com 
design to a standard optimization problem. ${ }^{1}$ For competing sellers, there is no analog to the revelation principle, i.e. no tool to abstract away from strategic behavior by buyers: given (even direct revelation) mechanisms announced by sellers, how buyers select between sellers is determined only in equilibrium. Since a seller's revenue depends on how many and which buyers visit, his problem returns to being optimization with a fixed point subroutine.

Due to these two issues, literature on the problem of competing mechanism designers has had to find ways around solving the 'full' problem. A few paths have been followed previously.

- A simple space of mechanisms: In [Burguet and Sakovics 1999] sellers choose only the reserve of a second price mechanism. This circumvents the former issue by fiat and makes the latter issue easier since they show that the equilibrium choice of buyers is 'easy' to determine.

- Large Markets: An assumption that the market is large is easier to deal with, since changing one seller's strategy does not effect the rest of the market. See for example [McAfee 1993], [Peters and Severinov 1997] and [Virag 2007].

-Infinite Regress: Some authors have followed the route of explicitly solving the infinite regress problem and identifying the full set of feasible mechanisms sellers can offer (e.g. [Peters 2001], [Pavan and Calzolari 2009]). Calculating equilibria when sellers can choose from this full set of mechanisms has proven difficult.

For the rest of this letter we list some new results in this area. Section 2 discusses [Feldman et al. 2010], which studies an applied model motivated by Ad Exchanges where agents compete via a restricted class of mechanisms. In Section 3 we discuss [Pai 2009]. Here, we study duopoly competition among sellers. Sellers can announce mechanisms from a more complete set than [Burguet and Sakovics 1999], but are still restricted from conditioning their choice of mechanism on the other seller's choice. We conclude with passing mentions of related recent results, and some directions for future work.

\section{AUCTIONS WITH INTERMEDIARIES}

The literature we discussed above studies settings where competing sellers who each have some units of a good (supply) compete for buyers (demand). There may however be settings where the converse is true: sellers have a captive (if unknown) demand, but compete upstream (at a producer/ supplier) for supply.

[Feldman et al. 2010] studies such a setting motivated by the new real time Ad Exchanges promoted by the internet search majors. An exchange sells a spot (inventory) in real time via a mechanism. However, advertisers do not directly bid in the exchange- rather, they bid via intermediaries known as Ad Networks. The paper asks how an ad exchange might design its mechanism knowing that own-profit-motivated intermediaries are bidding in the exchange's mechanism.

\footnotetext{
${ }^{1}$ In fact for the classical case considered in [Myerson 1981] and several extensions where buyers have additional private information (e.g. budget constraints), the seller's problem can be reduced to a 'nice' linear program (feasible space forms a polymatroid). See, e.g., [Pai and Vohra 2009] for details.

ACM SIGecom Exchanges, Vol. 9, No. 1, June 2010.
} 
The model has buyers with private types who are each 'captive' to an intermediary. The exchange is constrained to announce a second price auction - it only sets the reserve price. Intermediaries then select a 'contingent' auction (also a second price mechanism) to run among its captive bidders, and run this contingent auction. They bid in the exchange as a function of these contingent auctions, and the winning intermediary then transfers the good to the winner of the contingent auction at the contingent price. ${ }^{2}$ Intermediaries therefore have a captive demand, and must design mechanisms in order to extract revenue from buyers and also win the good in the upstream auction. In this sense our intermediaries compete by mechanisms.

In this setting, the paper identifies the equilibrium among intermediaries as a function of the mechanism announced by the exchange. It shows that the unique symmetric equilibrium among intermediaries involves a mixed strategy. Analogous to the literature on double marginalization, the minimum reserve price in the support of the intermediaries' strategies is an appropriately computed markup on the reserve price of the exchange. A profit maximizing exchange must take into account how this equilibrium changes as a function of the mechanism the exchange announces. The paper characterizes the optimization problem of the exchange, and considers the comparative statics of the system as various parameters change: e.g. number of intermediaries, buyers per intermediary etc.

The result can be interpreted thusly: a key feature an exchange might provide to publishers (e.g. people with inventory) is a guidance on what reserve price to charge. If the exchange uses standard auction theory it will provide incorrect guidance. Intuitively it will systematically overestimate the optimal reserve since it does not take into account the markup that will be added on by own-profit motivated intermediaries. This paper suggests how to compute the 'correct' reserve price guidance, albeit in an idealized setting.

\section{COMPETING AUCTIONEERS}

One critique of the literature on oligopolistic competing auctioneers is that the few results that exist are in extremely restrictive settings. For example, as we mentioned earlier, [Burguet and Sakovics 1999] restricts sellers to announcing second price mechanisms. Several 'natural' mechanisms are ruled out by this assumption: for example posted prices. ${ }^{3}$ [Pai 2009] considers competing limited supply sellers who can offer a larger class of mechanisms. Sellers are allowed to offer any 'hierarchical allocation rule'- intuitively a mechanism that allots to types according to some announced priority on types, randomizing in the case of ties. Note that both

\footnotetext{
${ }^{2}$ Astute readers may note the similarity of this setting to the literature on bidding rings, e.g. [McAfee and McMillan 1992]. The key difference is that in the literature on bidding rings the center only serves a coordinating device to maximize the joint profit of the ring. In our setting, the intermediary is motivated by his own profit. Our techniques may shed some light, however, on auctions when there are multiple competing rings, and how to design auctions knowing that the buyers have organized into rings.

${ }^{3}$ Even sellers with limited supply often use posted prices- think Buy It Now prices on E-Bay. When there is limited supply, sellers honor posted prices by randomizing among buyers willing to buy at the post price if demand exceeds supply. In the case of E-Bay, this randomization is achieved by the random buyer arrival process.
} 
second price mechanisms and posted prices are included, as are any hybrid of the two. ${ }^{4}$ Buyers with private values and unit demand choose which seller to visit as a function of sellers' announcements.

In this setting, the paper considers a fundamental question: oligopolistic markets often lead to inefficiency. However there are two sources of inefficiency- withholding by the seller (too little trade) and misallocation by the seller (the wrong kind of trade). The former happens, for example, when the seller runs an auction with a reserve price, and none of the buyers meet the reserve. The latter happens, for example, when the seller announces a posted price, and demand exceeds supply. Our intuition, and the solution of the monopoly seller's problem, has caused us to expect only the former kind of inefficiency.

The paper shows that sufficient and 'almost' necessary conditions for there to exist an equilibrium where sellers do not misallocate is that the distribution of buyers' types satisfy two conditions. The first is the monotone hazard rate condition, which is standard in mechanism design since [Myerson 1981]. The second requires that the density of the distribution is decreasing in valuation. These two conditions work in opposite directions: the first requires that the tail is not shrinking too fast, the second that the tail is shrinking at an increasing rate. Hence, in a sense, one should expect an equilibrium to also have a misallocation inefficiency, since the intersection of these conditions is satisfied by a small set of distributions. In terms of predictions, the paper suggests that sellers will use mechanisms that have a posted price component. This is even when they are free to choose auctions, without any of the costs associated with running an actual auction.

The intuition at the heart of this paper is easy to explain. In a monopoly setting, buyers are captive to a seller. The monotone hazard rate is sufficient and almost necessary for the revenue maximizing mechanism to not misallocate the good. But when competition is present, a mechanism must both extract revenue from buyers who visit the seller and provide enough surplus to buyers so that they do in fact visit in equilibrium. When the decreasing density condition fails, misallocating in an interval of valuations where the condition fails can provide more surplus to higher value buyers than not. While misallocation might reduce revenue from the misallocated buyers (if the monotone hazard condition is satisfied)- it entices more high valuation buyers to visit.

\section{CONCLUSION}

In this short letter we presented some recent progress in settings where sellers compete by announcing mechanisms. By any measure, much more progress is needed before such competition can be said to be 'understood' the way we understand competition in prices or quantities (or for that matter monopolist mechanism design). Several basic theoretical questions are unanswered in such settings. To name a few, what are sellers' incentives to invest in producing/ buying the good, or what is the effect of entry of one more seller into the market place.

There is growing academic interest in industries where competition is in mechanisms, or at least more 'complicated' than price/quantity competition- search engines, airlines \& health insurance markets to name a few. One can hope that

\footnotetext{
${ }^{4}$ For example, consider auctions on E-Bay that have a (high) Buy It Now price.
} 
such interest spurs both theoretical and applied research in this area. In our opinion, however, technical breakthroughs are necessary for progress toward 'workhorse' models that can be used across the board. The key as we suggested earlier is an analog to the revelation principle. The revelation principle can be thought of as a tool for abstracting from strategic behavior of buyers (fixed points) and reducing the sellers' problem to a standard optimization problem. Is there an analog when there are competing mechanisms? Since such an analog may be too much to hope for in general, a more realistic question may be to ask what constraints must be placed on mechanisms sellers can offer for there to be such a principle. For example, the results of [Burguet and Sakovics 1999] hinge on the fact that when sellers only offer second price mechanisms, the visit decisions of buyers is easy to compute. When are these constraints realistic depictions of the competitive settings we wish to study?

\section{REFERENCES}

Burguet, R. And Sakovics, J. 1999. Imperfect competition in auction designs. International Economic Review 40, 1, 231-247.

Feldman, J., Mirrokni, V., Muthukrishnan, S., And Pai, M. 2010. Auctions with intermediaries. In Forthcoming in ACM-EC.

McAfee, R. 1993. Mechanism design by competing sellers. Econometrica 61, 6, 1281-1312.

McAfee, R. And McMillan, J. 1992. Bidding rings. The American Economic Review 82, 3, $579-599$

Myerson, R. 1981. Optimal auction design. Mathematics of operations research 6, 1, 58-73.

PAI, M. 2009. Competing auctioneers.

Pai, M. AND Vohra, R. 2009. Optimal auctions with financially constrained bidders. Tech. rep., Working Paper, 2008. Available at: http://www. kellogg. northwestern. edu/faculty/Vohra/ftp/LR3. pdf.

Pavan, A. and Calzolari, G. 2009. Truthful revelation mechanisms for simultaneous common agency games. forthcoming American Economic Journal: Microeconomics.

Peters, M. 2001. Common agency and the revelation principle. Econometrica 69, 5, 1349-1372.

Peters, M. and Severinov, S. 1997. Competition among sellers who offer auctions instead of prices. Journal of Economic Theory 75, 1, 141-179.

VIRAG, G. 2007. Buyer heterogeneity and competing mechanisms. Tech. rep., University of Rochester. 